\title{
Case Report - Fire-Needle Acupuncture Treatment In Skin Papillomas Located on the Neck
}

\author{
Jihe Zhu ${ }^{1}$, Blagica Arsovska ${ }^{1,2}$, Kristina Kozovska ${ }^{1,3}$ \\ ${ }^{1}$ Faculty of Medical Sciences, University GoceDelchev, Shtip, Republic of Macedonia; \\ ${ }^{2}$ Institute of Biology, Faculty of Natural Sciences and Mathematics, Skopje, Republic of Macedonia \\ ${ }^{3}$ Medicine Faculty, St. Cyril and Methodius University of Skopje, Republic of Macedonia \\ Corresponding author - Kristina Kozovska, 'OrceNikolov' 155/5-2 1000 Skopje, Macedonia
}

\begin{abstract}
Skin papillomas are noncancerous and outwardgrowing lumps that doesn't spread and are not aggressive. Papillomas are also known as verruca or warts when they reach to the skin. The surface in which papilloma occurs in is called epithelia. The most common cause for papillomas is Human papillomavirus $(H P V)$. There are more than 100 different HPV types. In Traditional Chinese Medicine (TCM) warts are considered to occur because of liver weakness, caused by wind heat invasion. The treated patient is a woman who was admitted to the clinic for removal of multiple skin warts located on the neck. Only one treatment was made with fire-needle acupuncture. Acupuncture was done directly on the warts with a few punctures on one wart. Treatment was with duration of 5-10 minutes. After the treatment, the skin needed one week to heal and the warts to fall off. After one week all warts were gone. Acupuncture whether done with hot or cold needles, can significantly improve people's health, boost theimmunity, remove viral infections, enhance the blood and Qi flow, and especially when done properly by a good trained doctor the positive results are inevitable.
\end{abstract}

Keywords - traditional Chinese medicine, acupuncture, treatment, papilloma, hpv

\section{INTRODUCTION}

Skin papillomas are noncancerous and outwardgrowing lumps that doesn't spread and are not aggressive. Papillomas are also known as verruca or warts when they reach to the skin. The surface in which papilloma occurs in is called epithelia. Most of them don't produce any symptoms beyond irritation and cause more psychological symptoms like worry and impact on the self-esteem due to their appearance. People who have a weakened immune system are at increased risk for warts. The virus grows in warm and damp areas, therefore most common locations where warts can be easily picked up are pools, locker rooms or shower stalls. The incubation period takes approximately 2 to 6 months. [1] [2] [3]

The most common cause for papillomas is Human papillomavirus (HPV). The main symptom of HPV infection is triggering excessive growth of skin cells, which make the affected area to look thickened, rough and hard or in some cases some papillomas are flat and smooth. Papillomas can appear on any part of the body, but they most commonly appear on the feet and hands. There are more than 100 different HPV types. In women, HPV infections are responsible for more than $50 \%$ of the infection-linked cancers. While there are links between HPV and cancer, especially cervical cancer, researchers believe that it takes between 10 and 30 years for HPV to develop into a malignant condition. HPV warts are classified in two types: low-risk and high-risk warts. Both types can cause growth of abnormal cells, but only the high-risk warts can lead to cancer because of their encoded E7 protein which can immortalize the human epithelial cells. [1] [2] [4]

The Western medicine treatment options are curettage, laser surgery, cryotherapy, excision, applying liquid nitrogen or some drug therapy. [1]

In Traditional Chinese Medicine (TCM) warts are considered to occur because of liver weakness, caused by wind heat invasion. Acupuncture as part of the TCM can be used in the treatment of HPV warts. The efficaciousness of acupuncture is due to its effect on the nervous system, due to the neuromodulation of the immune system in the intervening in the pathogeneses of infectious and inflammatory skin conditions, such as HPV, acne, urticarial and dermatitis. [5]

\section{CASE REPORT}

The treated patient is a woman who was admitted to the clinic for removal of multiple skin warts located on the neck. Treatment were made in a clinic for Traditional Chinese Medicine and acupuncture in Skopje, North 
Macedonia by a doctor specialist in acupuncture. Only one treatment was made with fire-needle acupuncture. Acupuncture was done directly on the warts with a few (3 to 5) punctures on one wart. The treatment was with duration of 5-10 minutes. After the treatment, the skin needed one week to heal and the warts to fall off. After one week all warts were cleared.

On picture 1 is shown the fire-needleused in the treatment.

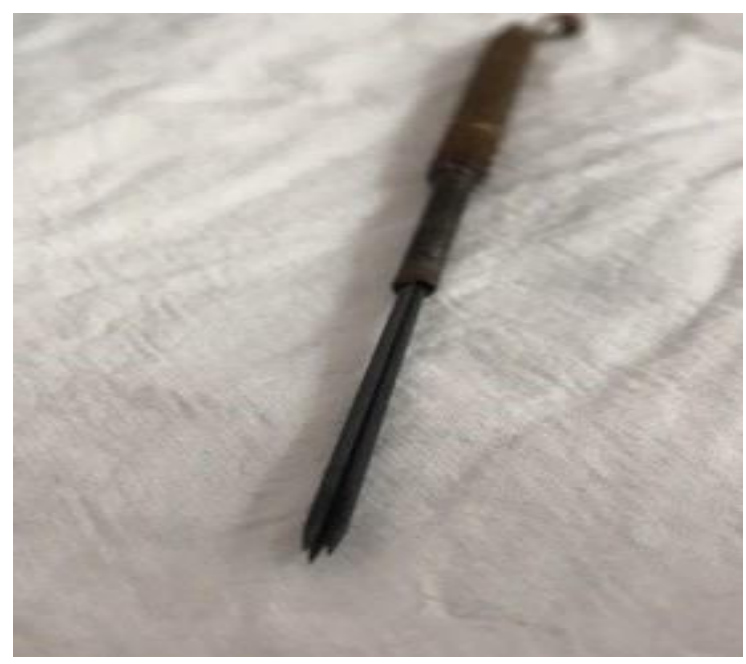

Picture 1. Fire-needle

On picture 2 is shown the patient's skin condition before

the

treatment

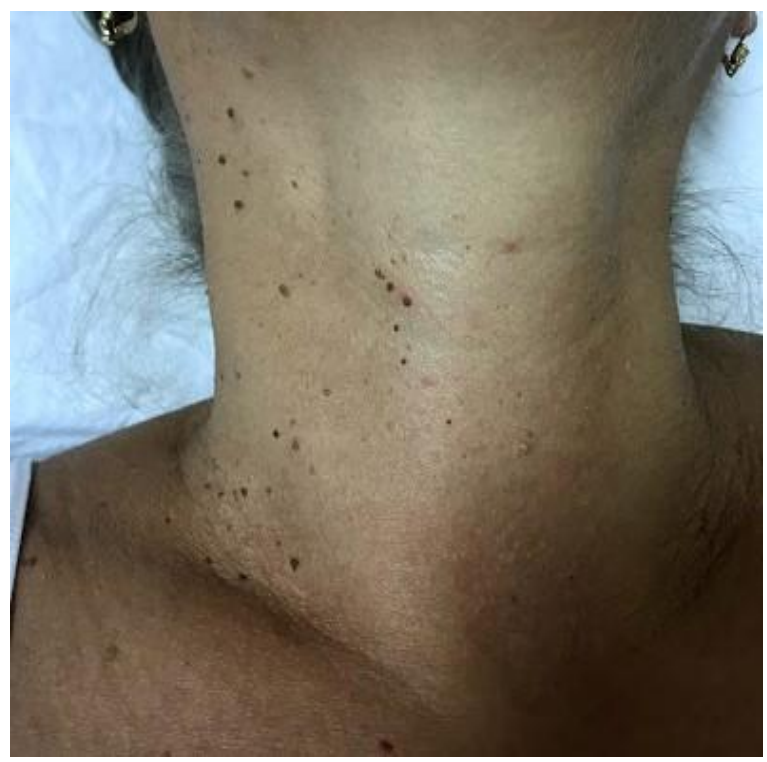

On picture 3 and 4 is shown the patient during the treatment and the performance on the fire-needle

Picture 3. Heating of the needle
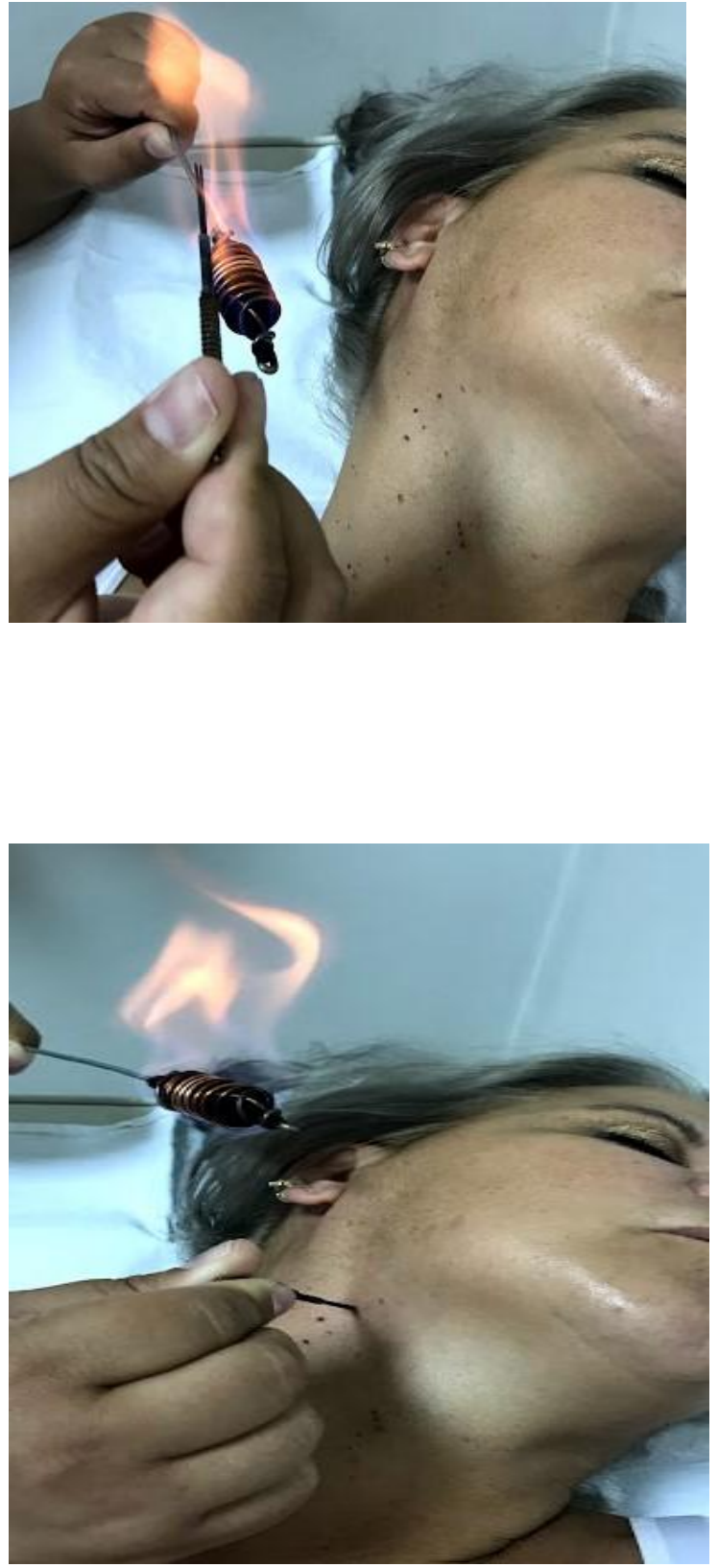

Picture 4. Puncturing the wart

Picture 2. Before treatment 
On picture 5 is shown the patient's skin condition after one week of the treatment.

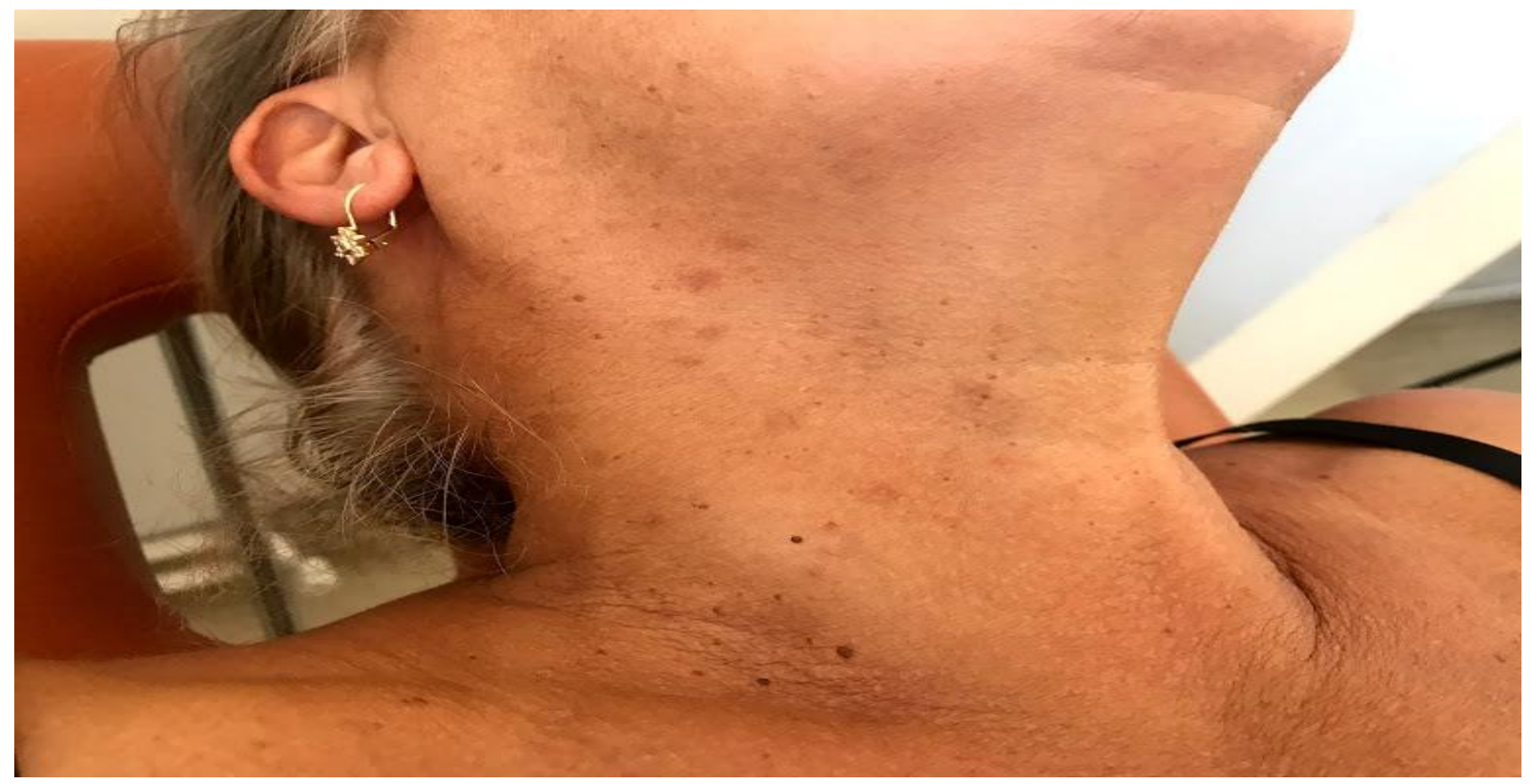

Picture 5. After the treatment

Filiform warts are the ones that grow around the face area, the eyes, mouth and nose. These warts grow very fast, have a sharp appearance and look like threads. These warts are usually painless and brown, pink, yellow or flesh-colored. [2]

TCM suggest strengthening and supporting the immune system with good nutrition and herbal treatment in order to cure the persistent HPV infection. All the systems in the body need to be in balance in order to fight the infection. [6]

Despite the fire-needle acupuncture, the treatment can be done with cold (normal) needle which involves insertion of the needles into the meridian points on the body. In a study done for warts removal, $1 / 3$ of the people who received weekly acupuncture treatments for 30 minutes in a 4 weeks period, experienced complete removal of the persistent warts compared to those patients who received placebo treatment. During the 3month follow up period there was no recurrence of the condition. For such skin condition, the immuneboosting properties of the acupuncture treatment are most responsible for these benefits. [2]

In other case was reported that with acupuncture treatment was cleared a wart in a HIV patient with a chronic HPV that was resistant to cryotherapy.The patient have done 58 acupuncture treatments in a period of 19 months. [5]
In a study for cervical cancer and genital warts, it was concluded that TCM improves the clinical index in the treatment as a results of its regulation of the cell apoptosis, immune function, the viral gene translation and transcription and cell signal transformation pathways. [7]

\section{CONCLUSION}

Acupuncture whether done with hot or cold needles, can significantly improve patient's health, boost the immunity, remove viral infections, enhance the blood and Qi flow, and especially when done properly by a good trained doctor the positive results are inevitable.

\section{References}

[1] MacGill M; Should I worry about a papilloma?; 2018 [www.medicalnewstoday.com]

[2] Davis PC; Types of Warts: Symptoms, Removal, and Treatment; 2018 [www.onhealth.com]

[3] Mannarini I et al.; Human Papilloma Virus (HPV) in head and neck region: review of literature; ActaOtorhinolaryngol Ital. 2009 Jun; 29(3): 119-126.

[4] Goldstein GB et al.; Cutaneous warts (common, plantar, and flat warts); 2019 [www.uptodate.com]

[5] Healthcare Medicine Institute; Acupuncture Clears Skin Conditions; 2015 [www.healthcmi.com]

[6] Wu AL; HPV: Awareness, prevention and treatment; 2015 [tcm-shanghai.com]

[7] Lin $\mathrm{J}$ et al.; Traditional Chinese medicine for human papillomavirus (HPV) infections: A systematic review; BioScience Trends. 2017; 11(3):267-273 